ARTICLE

\title{
A mechanically adaptive hydrogel with a reconfigurable network consisting entirely of inorganic nanosheets and water
}

Koki Sano (10 ${ }^{1 凶}$, Naoki Igarashi, ${ }^{1,2}$, Yasuo Ebina (i] ${ }^{3}$, Takayoshi Sasaki (i] ${ }^{3}$, Takaaki Hikima ${ }^{4}$, Takuzo Aida (i) ${ }^{1,2 凶}$ \& Yasuhiro Ishida (1D ${ }^{1 凶}$

Although various biomimetic soft materials that display structural hierarchies and stimuli responsiveness have been developed from organic materials, the creation of their counterparts consisting entirely of inorganic materials presents an attractive challenge, as the properties of such materials generally differ from those of living organisms. Here, we have developed a hydrogel consisting of inorganic nanosheets ( $14 \mathrm{wt} \%$ ) and water ( $86 \mathrm{wt} \%$ ) that undergoes thermally induced reversible and abrupt changes in its internal structure and mechanical elasticity (23-fold). At room temperature, the nanosheets in water electrostatically repel one another and self-assemble into a long-periodic lamellar architecture with mutually restricted mobility, forming a physical hydrogel. Upon heating above $55^{\circ} \mathrm{C}$, the electrostatic repulsion is overcome by competing van der Waals attraction, and the nanosheets rearrange into an interconnected 3D network of another hydrogel. By doping the gel with a photothermal-conversion agent, the gel-to-gel transition becomes operable spatiotemporally on photoirradiation.

\footnotetext{
${ }^{1}$ RIKEN Center for Emergent Matter Science, 2-1 Hirosawa, Wako, Saitama 351-0198, Japan. ${ }^{2}$ Department of Chemistry and Biotechnology, School of Engineering, The University of Tokyo, 7-3-1 Hongo, Bunkyo-ku, Tokyo 113-8656, Japan. ${ }^{3}$ National Institute for Materials Science, International Center for Materials Nanoarchitectonics, 1-1 Namiki, Tsukuba, Ibaraki 305-0044, Japan. ${ }^{4}$ RIKEN SPring-8 Center, 1-1-1 Kouto, Sayo, Hyogo 679-5198, Japan.

凶email: koki.sano@riken.jp; aida@macro.t.u-tokyo.ac.jp; y-ishida@riken.jp
} 
n scientific fiction and mythology, inorganic creatures often appear. These stories present us with a new challenge in materials science of how to produce life-like materials entirely from inorganic substances. This seems considerably difficult because living organisms usually consist of water-rich flexible solids with a structural hierarchy that are capable of responding to stimuli, unlike inorganic materials, which generally exhibit poor processability, low flexibility, and a lack of responsiveness. Indeed, previous studies on biomimetic soft materials have almost exclusively involved the use of organic constituents ${ }^{1-8}$, as typically represented by mechanically adaptive hydrogels based on thermoresponsive organic polymers such as $\operatorname{poly}(\mathrm{N}$-isopropylacrylamide $)^{7-13}$. However, if counterparts of these hydrogels consisting entirely of inorganic materials were to become available, they could expand the scope of materials science by adopting a complementary role to organic-based biomimetic soft materials in providing such characteristics as good mechanical properties, long-term durability, and low environmental burdens $^{14-16}$

For the creation of inorganic-based biomimetic soft materials, the most promising constituents are inorganic nanomaterials such as nanoparticles, nanofibers, or nanosheets ${ }^{17}$. When these inorganic materials self-assemble to form $3 \mathrm{D}$ architectures in water, they sometimes afford hydrogels ${ }^{18-25}$. In most cases, the inorganic nanomaterials aggregate through attractive interactions, such as van der Waals attraction or electrostatic attraction, to form interconnected $3 \mathrm{D}$ networks that can hold large quantities of water; these materials are referred to as attraction-dominant gels (Fig. 1e-g) $)^{18-22}$. However, inorganic nanomaterials can also participate in another type of self-assembly to produce a $3 \mathrm{D}$ architecture that is capable of exhibiting a gel-like behavior, although previous examples are limited to only a few examples on charged inorganic nanosheets ${ }^{23-25}$. If charged nanosheets exhibit a sufficiently strong mutual electrostatic repulsion, they can spontaneously self-assemble into a long-periodicity lamellar architecture in which their mobility is restricted. As a result, their aqueous dispersions can exhibit a gel-like behavior and such materials are referred to as repulsion-dominant gels (Fig. 1b-d).

Although these two types of inorganic-based hydrogel have been studied separately by using various nanomaterials, we conjectured that a single aqueous system containing charged nanosheets might be capable of reversibly forming both types of hydrogel through careful tuning of the intensity of the competing attractive and repulsive forces according to the Derjaguin-Landau-Verwey-Overbeek (DLVO) theory ${ }^{26,27}$. Such a hydrogel might be endowed with responsiveness to stimuli, despite its inorganic composition.

In this work, by adopting the above strategy, we succeeded in developing a stimuli-responsive hydrogel consisting of anionic nanosheets of titanate ${ }^{27-30}$ (TiNSs; $14 \mathrm{wt} \%$ ) and water (86 wt\%) that, depending on the temperature, can reversibly adopt one of two hydrogel states: a repulsion-dominant state or an attractiondominant state (Fig. 1). In this gel-to-gel transition driven by tuning of the electrostatic repulsion between TiNSs, the topology of the internal structure of the hydrogel changes abruptly (Fig. 2). Owing to the 2D shape of TiNSs, which prevents the possibility of entanglement, and the particular thermoresponsiveness of TiNSs, the gel-to-gel transition occurs rapidly with little hysteresis and without deterioration upon repetition (Fig. 3). Because of the abrupt topological reconfiguration of the internal structure of the material, the gel-to-gel transition is accompanied by a 23 -fold change in the hydrogel's mechanical elasticity, reminiscent of that of sea cucumbers (Fig. 4) 31,32 . Furthermore, by doping with a small number of gold nanoparticles that act as photothermal converters $^{33}$, the hydrogel can become responsive to photo stimuli ${ }^{33-36}$, permitting the gel-to-gel transition to occur in a spatiotemporally controlled manner (Fig. 5). Importantly, it is usually difficult to construct a gel that displays such a topological reconfiguration, even by using organic materials 37,38 .

\section{Results}

Preparation and characterization of a hydrogel consisting of titanate nanosheets in the repulsion-dominant state (TiNSGel $_{\text {Repuls }}$ ). The key to our achievement was the use of a particular nanosheet of titanate (TiNS) characterized by its ultra-thin $(0.75$ $\mathrm{nm}$ ) and extra-wide (several micrometers) dimensions (Fig. 1a and Supplementary Fig. 1$)^{27-30}$. TiNS is densely populated with negative charges $\left(1.5 \mathrm{C} \mathrm{m}^{-2}\right)$ that are surrounded by tetrabutylammonium countercations. By using TiNS as a constituent, a repulsion-dominant hydrogel, denoted as TiNS-Gel ${ }_{\text {Repuls, was }}$ prepared as follows. A $0.4 \mathrm{wt} \%$ aqueous dispersion of TiNS (30 $\mathrm{mL}$ ) was centrifuged at $20,000 \mathrm{~g}$ for $1 \mathrm{~h}$, so that the dispersion became segregated into a TiNS-free supernatant $(\sim 29 \mathrm{~mL})$ and a water-rich sediment $([$ TiNS $]=14 \mathrm{wt} \% ; 0.86 \mathrm{~mL})$. In this sediment, TiNSs were confined close to one another so that their mutual electrostatic repulsion became strong enough to restrict their mobility. Consequently, the sediment existed as a physical gel (Fig. 1b-d; TiNS-Gel Repuls $)^{23-25}$ that could be isolated from the supernatant and removed from the centrifugation tube. Upon

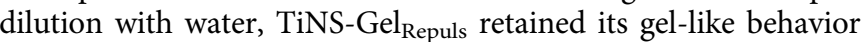
up to a TiNS concentration of $8 \mathrm{wt} \%$, but turned into a sol on further dilution (Supplementary Fig. 2).

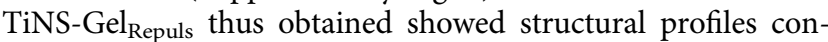
sistent with its supposed gelation mechanism as described above (Fig. 2a). In small-angle X-ray scattering (SAXS) measurements at $25^{\circ} \mathrm{C}$, TiNS-Gel $\mathrm{R}_{\text {Repuls }}$ exhibited multiple scattering peaks with $q$ value ratios of $1,1 / 2,1 / 3, \ldots$, and $1 / x$, suggesting the formation of a lamellar architecture of TiNSs with a uniform and large interlamellar distance of $11.8 \mathrm{~nm}$ (Fig. 2b). This distance is 16 times larger than the thickness of TiNS $(0.75 \mathrm{~nm})$, indicating that TiNSs were not in contact due to their mutual electrostatic repulsion (Fig. 1d). In a polarized optical microscopy (POM) study at $25^{\circ} \mathrm{C}$, TiNS-Gel Repuls $_{\text {exhibited a strong birefringence }}$ under crossed Nicols (Fig. 2c), which also confirmed the existence of a liquid-crystalline lamellar architecture in TiNS-Gel Repuls $_{\text {s }}$ (Fig. 1c). Furthermore, scanning electron microscopy (SEM) observations of a freeze-dried sample of TiNS-Gel Repuls $_{\text {showed }}$ the presence of a layered arrangement of TiNSs (Fig. 2d), probably originating from a lamellar architecture of TiNSs before freeze drying.

Preparation and characterization of a hydrogel consisting of titanate nanosheets in the attraction-dominant state (TiNSGel $_{\text {Attract }}$ ). We previously reported that the electrostatic repulsion between TiNSs becomes attenuated upon raising the temperature $^{27}$. Although this tendency had been observed in aqueous TiNS dispersions, we conjectured that a similar phenomenon might occur in a hydrogel of TiNSs (TiNS-Gel). Indeed, when TiNS-Gel $l_{\text {Repuls }}$ was heated from 25 to $90^{\circ} \mathrm{C}$, it changed into another type of hydrogel with a different internal structure and different physical properties (Fig. 2e-h). Throughout this transition, exudation of water from the hydrogel was not observed at all, suggesting that the volume of the hydrogel remained. Because the formation of this hydrogel was dominated by attractive forces between TiNSs, as later discussed (see Fig. 6a-c below), this hydrogel is denoted as TiNS-Gel Attract $_{\text {. }}$

In the SAXS profiles of TiNS-Gel ${ }_{\text {Attract }}$ at $90^{\circ} \mathrm{C}$, the sets of diffractions due to the lamellar architecture of TiNSs with a

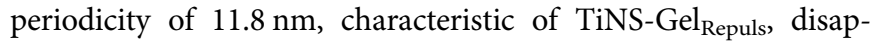
peared, while a single diffraction corresponding to a periodicity of $2.6 \mathrm{~nm}$ emerged (Fig. $2 \mathrm{f}$ ). The new periodicity was similar to that 


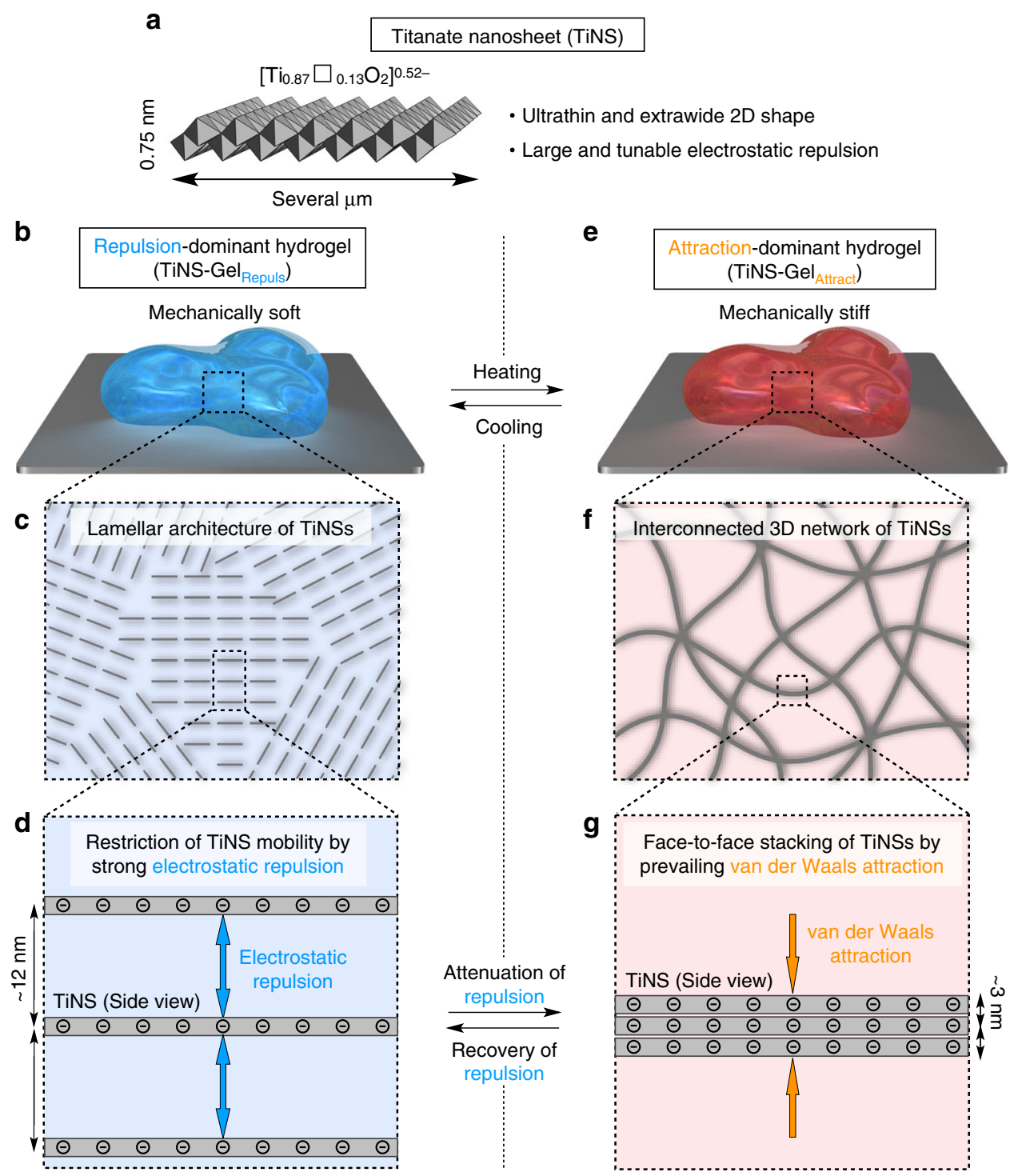

Fig. 1 Thermoresponsive hydrogel consisting of inorganic nanosheets (titanate nanosheet; TiNS) and water. a A schematic illustration of unilamellar titanate (IV) nanosheet (TiNS). Countercations are omitted for clarity. Open square indicates vacant sites. $\mathbf{b}-\mathbf{g}$ Schematic illustrations of the hydrogel of

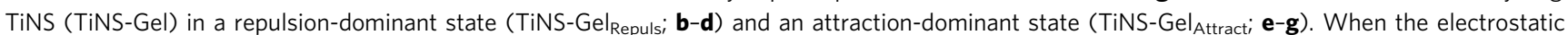
repulsion between TiNSs in an aqueous dispersion is strong enough, TiNSs spontaneously self-assemble into a long-periodicity lamellar architecture (c) in which their mobility is mutually restricted (d). As a result, their aqueous dispersion can exhibit a gel-like behavior, denoted as TiNS-Gel Repuls. When TiNS$\mathrm{Gel}_{\text {Repuls }}$ is heated above a critical temperature, the electrostatic repulsion becomes weaker than the competing van der Waals attraction, so that TiNSs

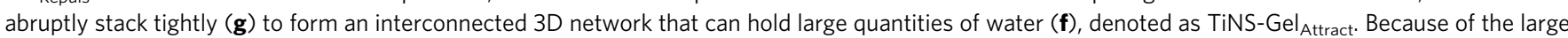

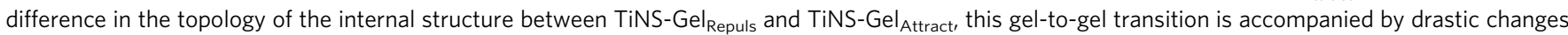
in the optical and mechanical properties.

of aggregates of TiNSs prepared by drying an aqueous TiNS dispersion $([\mathrm{TiNS}]=0.4 \mathrm{wt} \%)$ at a relative humidity of $95 \%$ for $12 \mathrm{~h}(d=\sim 2.7 \mathrm{~nm})^{28}$. By taking account of the SAXS

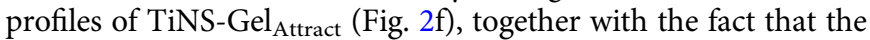
transition from TiNS-Gel Repuls $_{\text {to }}$ TiNS-Gel Attract $_{\text {proceeded }}$ without a change in the gel volume, the internal structure of TiNS-Gel Attract $_{\text {is }}$ considered to consist of an interconnected 3D network formed by partial stacking of TiNSs (Fig. 1f, g). In POM observations at $90^{\circ} \mathrm{C}$, TiNS-Gel Attract $_{\text {did not show }}$ any birefringence (Fig. $2 \mathrm{~g}$ ), indicating that its internal $3 \mathrm{D}$ structure is macroscopically isotropic, unlike that of TiNS$\mathrm{Gel}_{\text {Repuls. }}$ SEM observations of a freeze-dried sample of

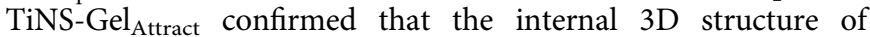
TiNS-Gel Attract $_{\text {was }}$ markedly different from that of TiNS$\mathrm{Gel}_{\text {Repuls }}$ (Fig. 2h).
Thermoresponsive transition between TiNS-Gel Repuls and

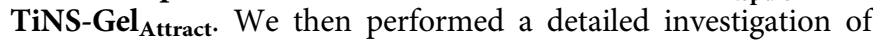
how the transition between TiNS-Gel ${ }_{\text {Repuls }}$ and TiNS-Gel $\mathrm{Attract}_{\text {}}$ occurs. First, we examined TiNS-Gel ([TiNS $]=14 \mathrm{wt} \%$ ) by differential scanning calorimetry at temperatures between 25 and $90{ }^{\circ} \mathrm{C}$ at a scanning rate of $1^{\circ} \mathrm{C} \mathrm{min}-1$ (Fig. 3a). A sharp single peak was observed at $\sim 55^{\circ} \mathrm{C}$ during both heating and cooling of the gel (Fig. 3b), confirming that the transition between TiNS-

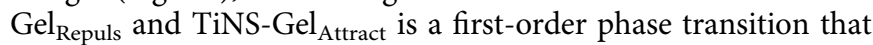
occurs at $\sim 55^{\circ} \mathrm{C}$.

To clarify how the internal structure changed in the gel-to-gel transition, we monitored the SAXS profiles of TiNS-Gel ([TiNS] $=14 \mathrm{wt} \%$ ) while performing a temperature sweep between 25 and $90{ }^{\circ} \mathrm{C}$ (Fig. 3c and Supplementary Fig. 3). As described above (Fig. 2b), TiNS-Gel $\mathrm{Repuls}_{\text {s }}$ at $25^{\circ} \mathrm{C}$ showed a set of diffractions 

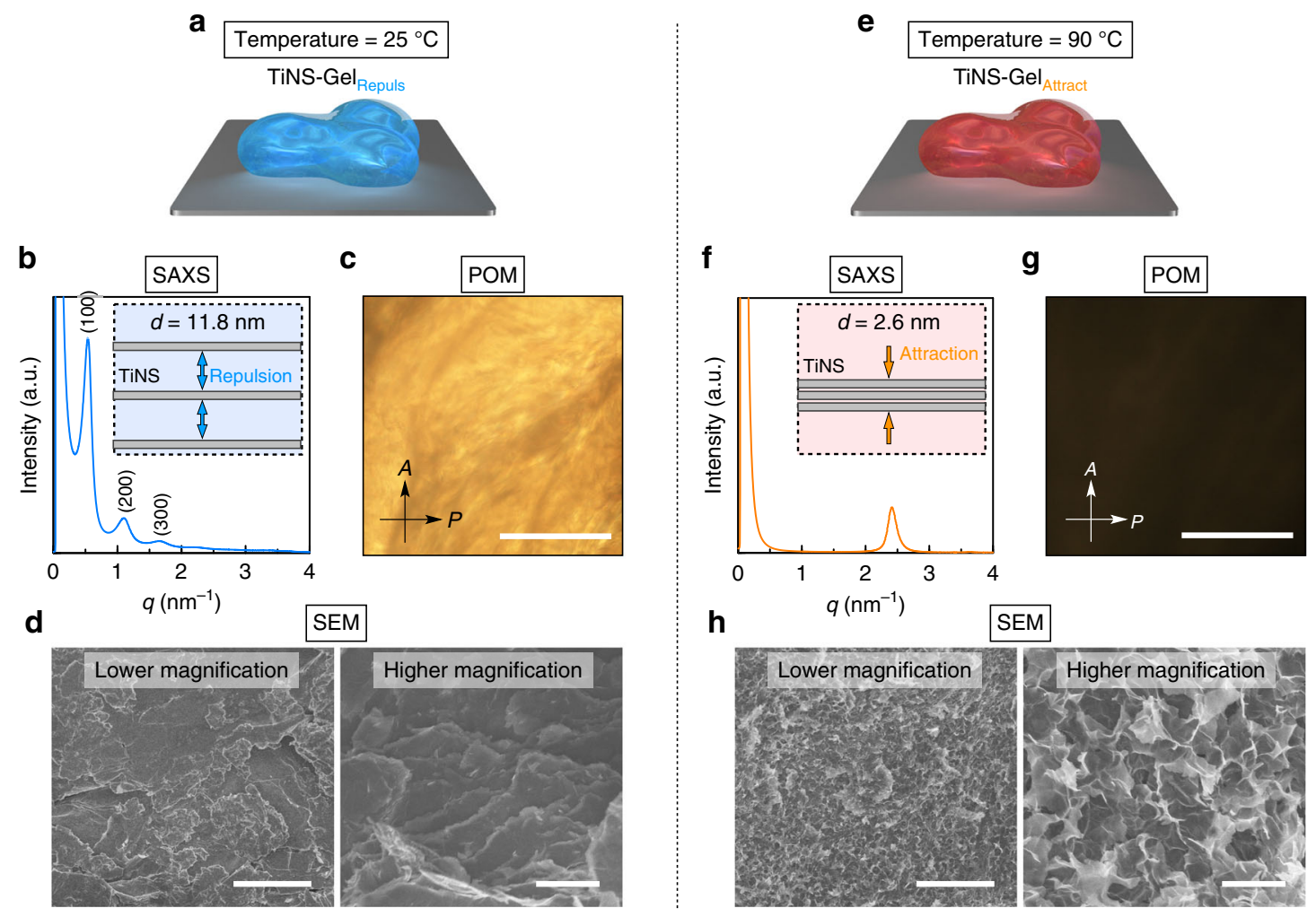

Fig. 2 Internal structures of TiNS-Gel $\mathbf{R e p u l s}_{\mathbf{s}}$ and TiNS-Gel $\mathbf{A t t r a c t}_{\mathbf{~}} \mathbf{a}, \mathbf{e}$ Schematic illustrations of the hydrogel of TiNS (TiNS-Gel) in a repulsion-dominant

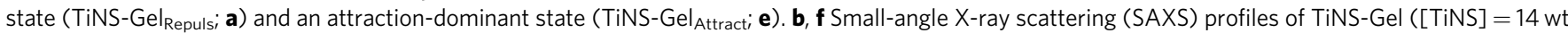
$\%)$ at $25^{\circ} \mathrm{C}(\mathbf{b})$ and $90^{\circ} \mathrm{C}(\mathbf{f})$. Insets indicate a schematic illustration of the nanosheet arrangements at a nanoscale level. c, $\mathbf{g}$ Polarized optical microscopy (POM) images under crossed Nicols of TiNS-Gel ([TiNS $]=14 \mathrm{wt} \%)$ at $25^{\circ} \mathrm{C}(\mathbf{c})$ and $90^{\circ} \mathrm{C}(\mathbf{g})$. $\mathbf{d}, \mathbf{h}$ Scanning electron microscopy $(\mathrm{SEM})$ images of freeze-dried samples of TiNS-Gel ([TiNS $]=14$ wt\%) prepared by rapid freezing from $25^{\circ} \mathrm{C}(\mathbf{d})$ and $90{ }^{\circ} \mathrm{C}(\mathbf{h})$ using liquid nitrogen. Scale bars: $1 \mathrm{~mm}(\mathbf{c}, \mathbf{g})$; $50 \mu \mathrm{m}$ (d, h, left); $10 \mu \mathrm{m}$ (d, h, right).

ascribable to a lamellar architecture of TiNSs with a periodicity of $11.8 \mathrm{~nm}$ (Fig. 1d). Upon heating from 25 to $50{ }^{\circ} \mathrm{C}$, this set of diffractions shifted gradually to the wider-angle region due to shrinkage of an interlamellar periodicity to $\sim 8 \mathrm{~nm}$ (Fig. $3 \mathrm{c}$ ). When the temperature reached $55^{\circ} \mathrm{C}$, the set of diffractions suddenly disappeared and a single diffraction corresponding to a periodicity of $\sim 3 \mathrm{~nm}$ emerged, indicating transition into TiNS$\mathrm{Gel}_{\text {Attract }}$. Upon further heating to $90^{\circ} \mathrm{C}$, the new diffraction peak shifted slightly to the wider-angle region possibly because of the heat-induced dehydration of countercations of TiNSs and/or TiNS surfaces ${ }^{39}$. In the cooling process from 90 to $25^{\circ} \mathrm{C}$, the reverse changes took place. Even when this heating/cooling cycle was repeated, the temperature-dependent SAXS profiles remained largely unchanged (Supplementary Fig. 3).

To investigate the rate of the gel-to-gel transition, we subjected TiNS-Gel Repuls $([\mathrm{TiNS}]=14 \mathrm{wt} \%)$ to an abrupt change in temperature from 25 to $90{ }^{\circ} \mathrm{C}$ (Fig. $3 \mathrm{~d}$ ) and we monitored the time-course changes in its birefringence by using POM under crossed Nicols. The initial bright POM image darkened within $2 \mathrm{~s}$ (Fig. 3e), confirming the rapidity of the transition from TiNS$\mathrm{Gel}_{\text {Repuls }}$ into TiNS-Gel $\mathrm{Attract}_{\text {(Fig. 3f). }}$.

To summarize, in response to a temperature change, the internal network topology of TiNS-Gel switches quickly and sharply within a narrow temperature range with almost no hysteresis or deterioration upon repetition. Although the gel-togel transition involves a marked topological reconfiguration of the internal structure, it proceeds with excellent speed and reversibility, probably because the $2 \mathrm{D}$ shape of TiNSs eliminates the possibility of entanglement.
Mechanical properties of the TiNS hydrogel. Sea cucumbers, upon external stimulation, can abruptly switch their mechanical properties by modulating their internal structures ${ }^{31,32}$. This phenomenon has attracted significant attention from both fundamental and practical viewpoints. We conjectured that our TiNS-Gel might be capable of mimicking this function, because its gel-to-gel transition proceeded with excellent rapidity and perfect reversibility, and involves drastic topological reconfiguration of the internal structure of the gel.

To confirm this possibility, we conducted rheological tests on TiNS-Gel $([\mathrm{TiNS}]=14 \mathrm{wt} \%)$ at 25 and $90^{\circ} \mathrm{C}$, where it exists as

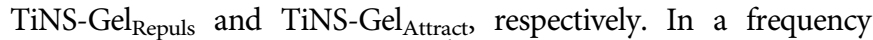
sweep (frequency $=0.1-10 \mathrm{rad} \mathrm{s}^{-1}$; strain $=0.1 \%$ ) at $25^{\circ} \mathrm{C}$, TiNS$\mathrm{Gel}_{\text {Repuls }}$ showed storage moduli $\left(G^{\prime}\right)$ that were higher than its loss moduli $\left(G^{\prime \prime}\right)$ over the entire frequency range, confirming its gel-like properties, where $G^{\prime}$ at a frequency of $1.0 \mathrm{rad} \mathrm{s}^{-1}$ was $26 \mathrm{kPa}$ (Fig. 4a). When the same setup was heated to $90^{\circ} \mathrm{C}$, the resultant TiNS-Gel Attract $_{\text {also showed a similar gel-like property with } G^{\prime}>G^{\prime \prime}}$ over the entire frequency region, but the $G^{\prime}$ value was more than 20 times larger than that of TiNS-Gel Repuls $\left(G^{\prime}=600 \mathrm{kPa}\right.$ at a frequency of $1.0 \mathrm{rad} \mathrm{s}^{-1}$; Fig. 4b). When a heating/cooling cycle between 25 and $90^{\circ} \mathrm{C}$ was repeated three times, the thermoresponsive changes in the $G^{\prime}$ and $G^{\prime \prime}$ values were barely affected (Fig. 4c), demonstrating the excellent reversibility and rapidity of this mechanical-property switching.

Compression tests also confirmed the ability of TiNS-Gel to change its mechanical properties in response to thermal stimuli. The Young's modulus of TiNS-Gel ([TiNS] $=14 \mathrm{wt} \%$ ) increased from 1.3 to $20 \mathrm{kPa}$ when it underwent a transition from TiNS- 
a

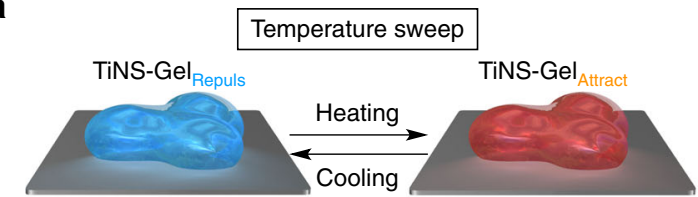

b

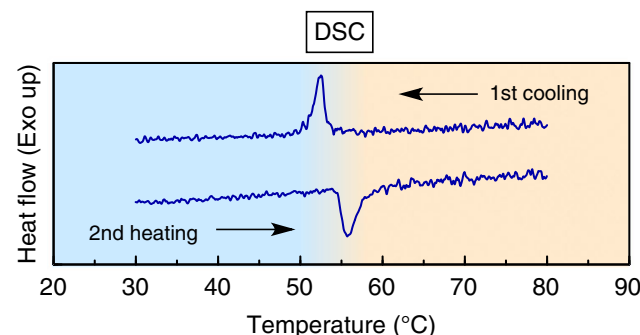

C

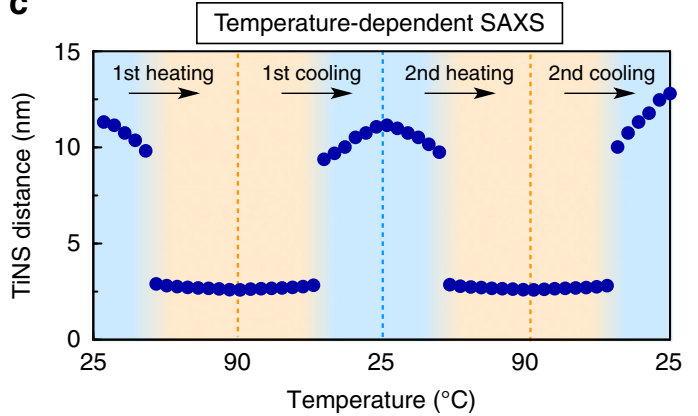

d

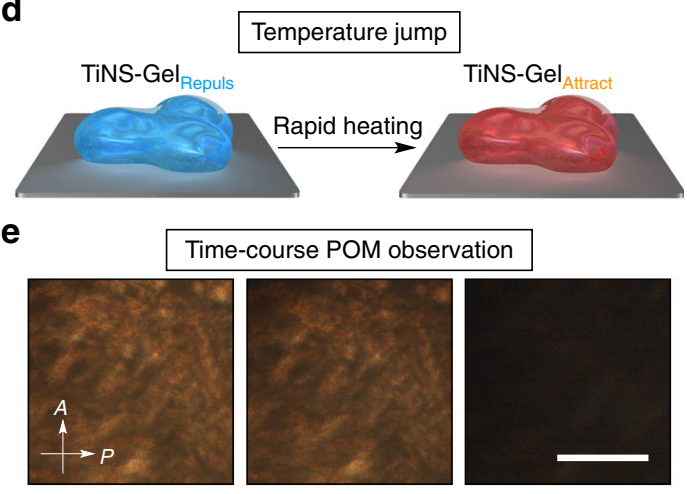

(i) $t=0$

(ii) $t=1.0$

(iii) $t=2.0$

Time after heating (s)

f

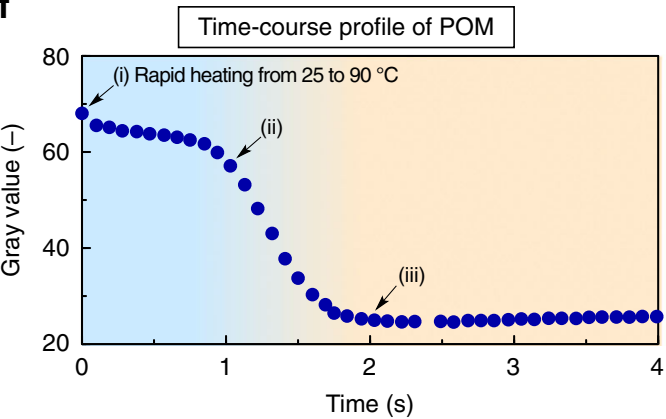

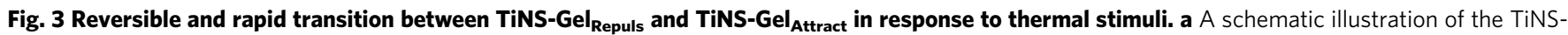
Gel behavior upon temperature sweep. $\mathbf{b}$ Differential scanning calorimetry (DSC) measurement of TiNS-Gel ([TiNS] $=14$ wt\%) between 25 and $90{ }^{\circ} \mathrm{C}$ at a rate of $1^{\circ} \mathrm{C} \mathrm{min}^{-1}$. c Changes in the TiNS distance of TiNS-Gel ([TiNS] $=14$ wt\%) upon repeated thermal scanning between 25 and $90{ }^{\circ} \mathrm{C}$. The TiNS distance was determined by SAXS measurements. d A schematic illustration of the TiNS-Gel behavior upon temperature jump. e, $\mathbf{f}$ Time-course POM images (e) and time-course plots of the gray value of the POM images (f) of TiNS-Gel ([TiNS] =14 wt\%) after an abrupt temperature jump from 25 to $90^{\circ} \mathrm{C}$. Scale bar: $1 \mathrm{~mm}(\mathbf{e})$.

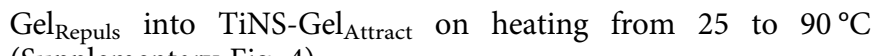
(Supplementary Fig. 4).

Spatiotemporal controllability of a TiNS hydrogel. The results reported above prompted us to attempt to produce a more life-like smart material from TiNS-Gel by expanding the range of stimuli capable of inducing the gel-to-gel transition. In particular, the use of light as a stimulus is an attractive option that should permit the gel-to-gel transition to occur in a spatiotemporally controlled manner (Fig. 5a), similar to some biological systems ${ }^{33-37}$. Because TiNS-Gel itself is thermoresponsive, it might be modified to produce a photoresponsive version by the addition of an appropriate photothermal converter.

As a photothermal converter, we chose gold nanoparticles (AuNPs) because of their water dispersibility, compatibility with TiNSs, and excellent photothermal-conversion ability ${ }^{33}$. Thus, TiNS-Gel Repuls $([\mathrm{TiNS}]=14 \mathrm{wt} \%$ ) was doped with monodisperse AuNPs ([AuNP] $=0.05 \mathrm{wt} \%)$ with an average diameter of $17 \mathrm{~nm}$ (Fig. 5b, c). The mechanical properties and thermoresponsiveness of AuNP-doped TiNS-Gel were well preserved (Supplementary Fig. 5). Also, the photothermal-conversion ability of AuNPs was preserved throughout the hybridization; when AuNP-doped TiNS-Gel was irradiated with 445-nm laser light (power density $=5.6 \mathrm{~W} \mathrm{~cm}{ }^{-2}$ ), the temperature of the irradiated region increased from 25 to $82{ }^{\circ} \mathrm{C}$ within $40 \mathrm{~s}$, as monitored by a thermal-imaging camera (Fig. 5d, right). Because AuNP-free TiNS-Gel did not show any temperature change under the same photoirradiation condition (Fig. 5d, left), the present photothermal conversion can be ascribed to the effects of AuNPs exclusively. When the light was turned off, the irradiated region of AuNP-doped TiNS-Gel was air-cooled to $25^{\circ} \mathrm{C}$ within $60 \mathrm{~s}$. The light-on/light-off cycle could be repeated many times without detectable deterioration (Fig. 5e).

As we envisioned in Fig. 5a, POM observation of these temperature-changing processes showed that AuNP-doped TiNSGel underwent a transition between TiNS-Gel Repuls $_{\text {and TiNS- }}$ $\mathrm{Gel}_{\text {Attract }}$ rapidly, reversibly, and in a spatiotemporally controlled manner (Fig. 5f). On photoirradiation of TiNS-Gel Repuls $_{\text {with }} 445$ $\mathrm{nm}$ laser light (power density $=5.6 \mathrm{~W} \mathrm{~cm}^{-2}$; irradiated region $=$ $2 \times 4 \mathrm{~mm}$ ) for $40 \mathrm{~s}$, the irradiated region was selectively transformed into TiNS-Gel Attract $_{\text {with no birefringence, whereas }}$ upon ceasing photoirradiation, the irradiated region returned to TiNS-Gel $l_{\text {Repuls }}$ with a large birefringence within $4 \mathrm{~s}$ (Supplementary Movie 1). Detailed studies revealed that (i) the transition from TiNS-Gel ${ }_{\text {Repuls }}$ to TiNS-Gel Attract $_{\text {under this condition was }}$ finished within $\sim 2 \mathrm{~s}$ and (ii) the transition speed can be tuned by changing the light power density (Supplementary Fig. 6b) and AuNP concentration (Supplementary Fig. 6c). This dynamic structural change, which is highly controllable in both time and space, is reminiscent of the responsive behaviors of living organisms, despite the inorganic composition of TiNS-Gel.

Key parameters for TiNSs to form a mechanically adaptive hydrogel. Although many hydrogels consisting entirely of such inorganic nanomaterials as nanoparticles, nanofibers, or nanosheets have been developed, TiNS-Gel is the only known hydrogel that is capable of reversibly adopting one of two hydrogel states. To clarify which are the key factors underlying this property, we conducted systematic studies on the characteristic profiles of TiNSs in terms of (i) its large and tunable 
a
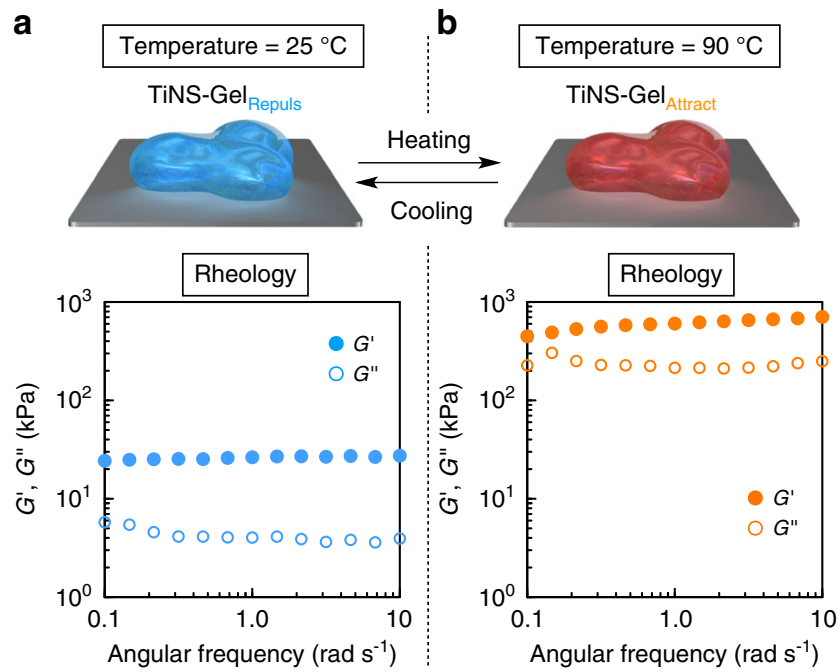

C

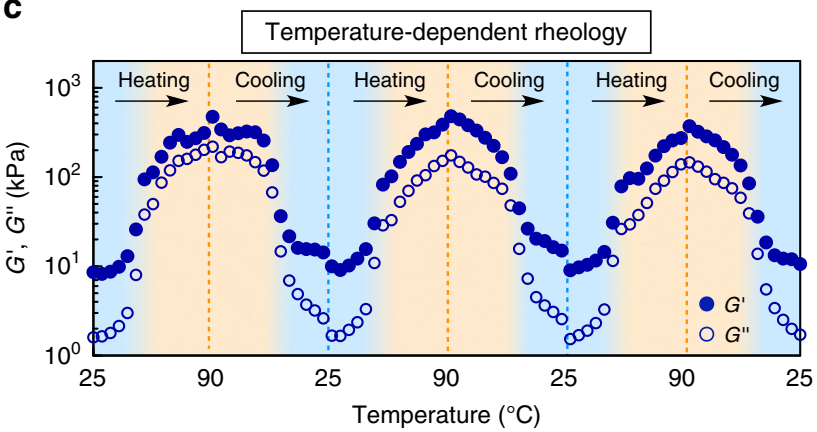

Fig. 4 Thermoresponsive switching of the mechanical properties of TiNSGel through the transition between TiNS-Gel $\mathbf{R e p u l s}_{\mathbf{s}}$ and TiNS-Gel $\mathbf{A t t r a c t}_{\text {- }}$ a, b Storage $\left(G^{\prime}\right)$ and loss $\left(G^{\prime \prime}\right)$ moduli on frequency sweep (0.1-10 rad s$^{-1}$ ) at a fixed strain $(0.1 \%)$ of TiNS-Gel ([TiNS $]=14 \mathrm{wt} \%)$ with keeping the temperature at $25^{\circ} \mathrm{C}(\mathbf{a})$ and $90^{\circ} \mathrm{C}(\mathbf{b})$. c Changes in the $G^{\prime}$ and $G^{\prime \prime}$ values at a fixed frequency $\left(1 \mathrm{rad} \mathrm{s}^{-1}\right)$ and a strain $(0.1 \%)$ of TiNS-Gel ([TiNS] $=$ $14 \mathrm{wt} \%$ ) upon repeated thermal scanning between 25 and $90^{\circ} \mathrm{C}$.

electrostatic repulsion and (ii) the ultra-thin and extra-wide 2D shapes of its nanosheets, as described below.

TiNSs carry a dense array of negative charges $\left(1.5 \mathrm{C} \mathrm{m}^{-2}\right)$ and they intrinsically show a large mutual electrostatic repulsion, which is essential in permitting the formation of TiNS-Gel $l_{\text {Repuls }}$ (Fig. 1b-d). For the reversible transition between TiNS-Gel Repuls and TiNS-Gel ${ }_{\text {Atract }}$, the electrostatic repulsion should be tunable (Fig. 1e-g) ${ }^{26,27}$. We found that the required tunability was provided by the thermoresponsive behavior of the TiNS surfaces, where tetrabutylammonium countercations $\left(\mathrm{T}^{+}\right)$and protons $\left(\mathrm{H}^{+}\right)$form ion pairs with surface anions $\left(\mathrm{Ti}-\mathrm{O}^{-}\right)$, as shown in Fig. 6a. When an aqueous dispersion of TiNSs is heated from 25 to $90^{\circ} \mathrm{C}$, the countercations $\mathrm{T}^{+}$, which are originally trapped by Ti- ${ }^{-}$on TiNSs, are replaced with $\mathrm{H}^{+}$from bulk water. Because of this thermally induced $\mathrm{T}^{+} / \mathrm{H}^{+}$replacement, the concentration of free $\mathrm{T}^{+}$ions increases from $0.25 \mathrm{mM}$ (at $25^{\circ} \mathrm{C}$ ) to $0.55 \mathrm{mM}$ (at $90^{\circ} \mathrm{C}$ ), as confirmed by ion conductometry (Fig. $6 \mathrm{~b}$, red). The thermally induced $\mathrm{T}^{+} / \mathrm{H}^{+}$replacement also causes neutralization of the negative charges on $\mathrm{Ti}^{-} \mathrm{O}^{-}$on TiNSs, thereby lowering the absolute value of the TiNS surface potential from $-60 \mathrm{mV}$ (at $25^{\circ} \mathrm{C}$ ) to $-36 \mathrm{mV}$ (at $70^{\circ} \mathrm{C}$ ), as confirmed by measurements of the zeta potential (Fig. 6c, red). According to the DLVO theory ${ }^{26,27}$, both of these heat-induced changes should contribute to an attenuation of the electrostatic repulsion between TiNSs, thereby explaining the mechanism of the transition from TiNS-

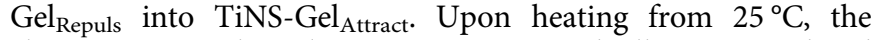
electrostatic repulsion between TiNSs is gradually attenuated and eventually, at $\sim 55^{\circ} \mathrm{C}$, becomes weaker than the competing van der Waals attraction, so that TiNSs abruptly stack tightly to form an interconnected 3D network, resulting in a transition from

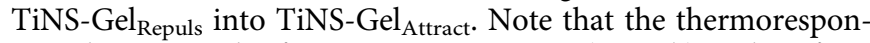
sive changes in the free-ion concentration (Fig. 6b) and surfacepotential intensity (Fig. 6c) are reversible and show little hysteresis, which is consistent with the reversible and littlehysteresis nature of the transition between TiNS-Gel Repuls $_{\text {and }}$ and TiNS-Gel $_{\text {Attract, }}$ as confirmed by the SAXS (Fig. 3c) and rheological measurements (Fig. 4c).

To clarify the roles of another key property of TiNSs, their ultra-thin and extra-wide 2D shape, we constructed a thermal phase diagram of TiNSs dispersed in water $([\mathrm{TiNS}]=14 \mathrm{wt} \%)$ with systematic changes in the size of TiNSs (Fig. 6d). Aqueous dispersions of TiNSs of various sizes were prepared by sonicating an as-synthesized aqueous TiNS dispersion ([TiNS $]=0.4 \mathrm{wt} \%$; original hydrodynamic size $=1.9 \mu \mathrm{m}$ ). The resulting TiNS dispersions were then concentrated to $[\mathrm{TiNS}]=14 \mathrm{wt} \%$ by centrifugation as described above. When the size of TiNSs was sufficiently large (hydrodynamic size $=1.2-1.9 \mu \mathrm{m}$ ), the TiNS dispersion formed TiNS-Gel Repuls $_{\text {in }}$ in the low-temperature region below $55^{\circ} \mathrm{C}$ (Fig. $6 \mathrm{~d}, \# 1, \# 2, \# 5$, and \#6) and TiNS-Gel $\mathrm{Attract}_{\text {in }}$ the high-temperature region above $55^{\circ} \mathrm{C}$ (Fig. $6 \mathrm{~d}$, \#3, \#4, \#7, and \#8). However, when the size of TiNSs was moderate (hydrodynamic size $=0.18-0.47 \mu \mathrm{m}$ ), the TiNS dispersion did not form TiNS$\mathrm{Gel}_{\text {Repuls }}$ in the low-temperature region but, instead, existed in a sol state (Fig. 6d, \#9, \#10, \#13, and \#14), probably because a reduction in the size of TiNSs enhanced their mobility, even though a strong electrostatic repulsion between TiNSs was present. In consistency with this, our previous report ${ }^{28}$ on an aqueous dispersion of TiNSs with relatively small size $(<\sim 1 \mu \mathrm{m})$ showed a lower tendency of forming a lamellar architecture than that of this work. Meanwhile, in the high-temperature region, moderate-sized TiNSs (hydrodynamic size $=0.18-0.47 \mu \mathrm{m}$ ) were

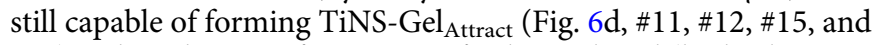
\#16). When the size of TiNSs was further reduced (hydrodynamic size $=0.11 \mu \mathrm{m}$ ), the TiNS dispersion did not form a hydrogel in either the low-temperature region or the high-temperature region (Fig. 6d, \#17-\#20), probably because the size of TiNS was so small that their heat-induced aggregation did not result in an interconnected 3D network, but instead resulted in the possible formation of densely packed columnar stacks of TiNSs ${ }^{40,41}$. Overall, a large lateral size of TiNSs is essential for the formation of TiNS-Gel Repuls and TiNS-Gel ${ }_{\text {Attract }}$, and the size of nanosheets required for the formation of TiNS-Gel Repuls $(\sim 1 \mu \mathrm{m})$ is much larger than that required for the formation of TiNS-Gel $\mathrm{Attract}_{\mathrm{A}}$ $(\sim 0.18 \mu \mathrm{m})$.

\section{Discussion}

We have developed a stimuli-responsive hydrogel (TiNS-Gel) consisting of inorganic constituents (14 wt\% TiNSs and $86 \mathrm{wt} \%$ water). In response to temperature changes, TiNS-Gel reversibly adopts one of two hydrogel states (TiNS-Gel Repuls $_{\text {or TiNS- }}$ $\mathrm{Gel}_{\text {Attract }}$ ) that have totally different internal structures: a lamellar architecture and an interconnected 3D network, respectively. The gel-to-gel transition proceeds within a narrow temperature range $\left(55 \pm 2{ }^{\circ} \mathrm{C}\right)$ and with excellent rapidity (within $\sim 2$ s) and perfect reversibility (little hysteresis and no deterioration upon repetition). The gel-to-gel transition was accompanied by marked changes in the optical properties (strong birefringence to no birefringence) and the mechanical properties $\left(G^{\prime}=26 \mathrm{kPa}\right.$ to $G^{\prime}=600 \mathrm{kPa}$ ) of the gel. Thus, the mechanical properties of TiNS-Gel changed considerably and rapidly as a result of 


\section{a $\quad$ AuNP-doped TiNS-Gel}
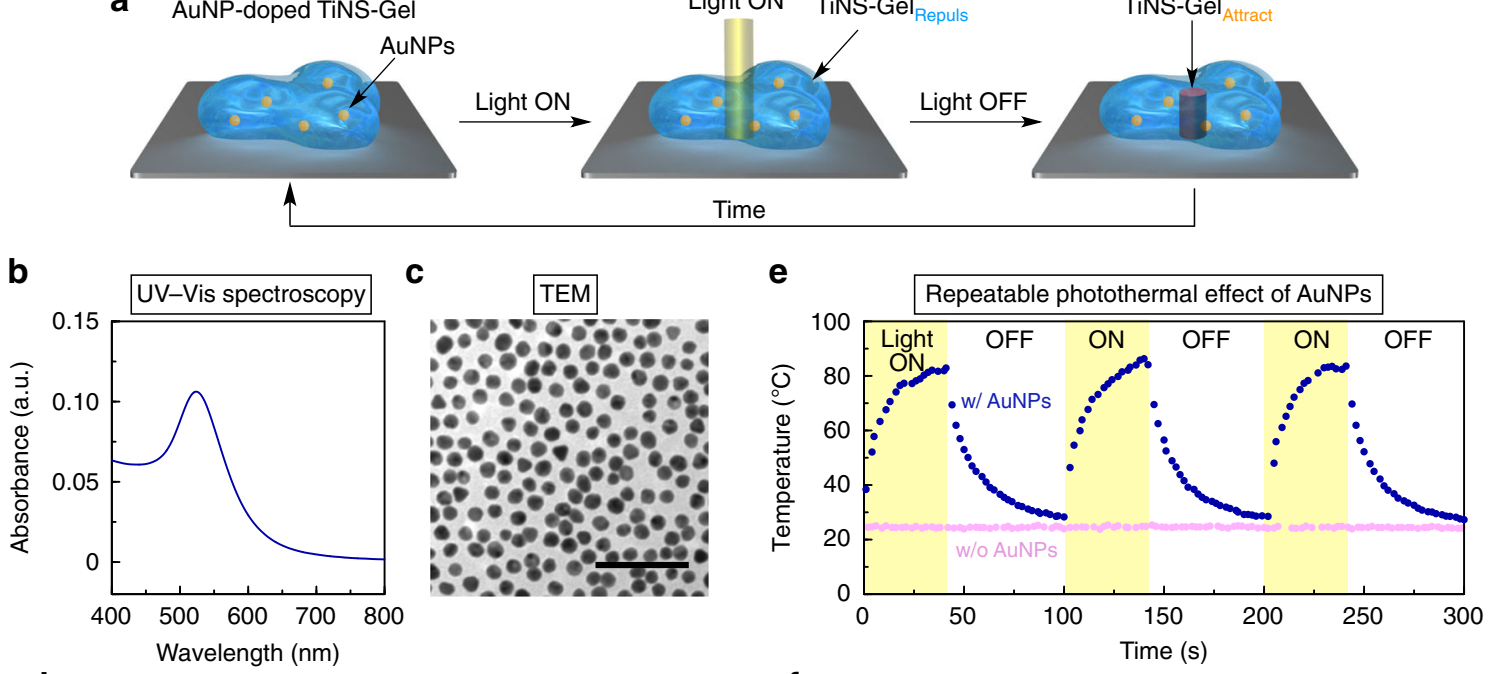

d
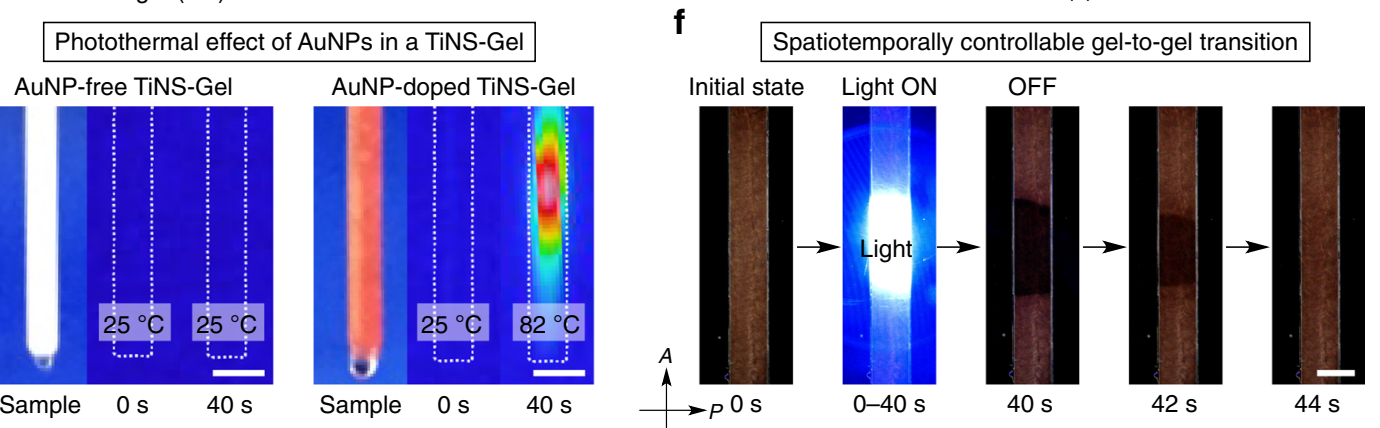

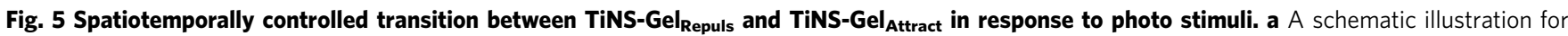
photoinduced gel-to-gel transition between TiNS-Gel Repuls and TiNS-Gel $\left.\right|_{\text {Attract }}$ b, $\mathbf{c}$ Characterization of gold nanoparticles (AuNPs; average diameter $=17$ $\mathrm{nm}$ ); UV-Vis absorption spectrum of an aqueous dispersion ([AuNP] $=0.005 \mathrm{wt} \% ; \mathbf{b}$ ) and transmission electron microscopy image (TEM image; c). d Photoinduced temperature changes upon irradiation with 445-nm laser light (power density $=5.6 \mathrm{~W} \mathrm{~cm}^{-2}$ ) of TiNS-Gel ([TiNS] $=14 \mathrm{wt} \%$ ) without (left) and with (right) $0.05 \mathrm{wt} \%$ AuNPs. Pictures of the samples in a 1.5-mm diameter glass capillary (left) and their thermal-imaging camera images before (mid) and after (right) 40-s light irradiation. e Time-course temperature changes of TiNS-Gel ([TiNS] =14 wt\%) without (pink dots) and with (navy dots) 0.05 wt \% AuNPs upon repeated turning on and off of the 445-nm laser light, which were monitored by a thermal-imaging camera. $\mathbf{f}$ Photoinduced changes in POM images under crossed Nicols of TiNS-Gel doped with AuNPs ([TiNS $]=14$ wt\%; $[$ AuNP] $=0.05$ wt\%). When TiNS-Gel Repuls in a 0.2-mm-thick glass container was irradiated with the $445-\mathrm{nm}$ laser light (irradiated region $=2 \times 4 \mathrm{~mm}$ ) for $40 \mathrm{~s}$, the irradiated region was selectively transformed into TiNS-Gel ${ }_{\text {Attract }}$ with no birefringence. Upon ceasing photoirradiation, the irradiated region returned to TiNS-Gel Repuls with a large birefringence within $4 \mathrm{~s}$. This cycle could be repeated many times without deterioration (Supplementary Movie 1). Scale bars: $100 \mathrm{~nm}$ (c); $2 \mathrm{~mm}$ (d, f).

topological reconfiguration of its internal structure, in a manner reminiscent of sea cucumbers. By doping with gold nanoparticles as photothermal converters, the gel-to-gel transition could be induced by laser irradiation in a spatiotemporally controlled manner. To the best of our knowledge, no such smart material has previously been produced from inorganic materials exclusively.

The keys to this achievement are the characteristic profiles of TiNSs, which have an ultra-thin and extra-wide 2D shape and large and tunable electrostatic repulsion. Their shape endows TiNSs with the ability to form self-assembled 3D structures that contain abundant water efficiently, while their electrostatic properties permit the topology of these $3 \mathrm{D}$ structures to change in response to thermal stimuli. The $2 \mathrm{D}$ shape of TiNSs, which eliminates the possibility of entanglement, may be the reason for the excellent rapidity and reversibility of the thermal response of TiNS-Gel. These properties appear to be superior to those of conventional hydrogels based on 1D materials such as organic polymers or nanofibers ${ }^{1-13}$. Although poly $(N$-isopropylacrylamide) and related organic polymers have long been the components of choice for smart soft materials ${ }^{42,43}$, we have demonstrated that TiNS can serve as an alternative to such organic polymers. This work will surely expand the scope of adaptive materials of the next generation, possibly even leading to the creation of 'inorganic life'.

\section{Methods}

Preparation of the hydrogel of TiNS (TiNS-Gel). In a typical procedure, an aqueous dispersion $(30 \mathrm{~mL})$ of TiNS $\left([\mathrm{TiNS}]=0.4 \mathrm{wt} \%,\left[\left(\mathrm{C}_{4} \mathrm{H}_{9}\right){ }_{4} \mathrm{~N}^{+} \mathrm{OH}^{-}\right]=\right.$

$\sim 90 \mathrm{mM}$ ) in a $50-\mathrm{mL}$ centrifuge tube was centrifuged at $20,000 \mathrm{~g}$ for $1 \mathrm{~h}$. From the resultant segregated mixture, the supernatant $(\sim 29 \mathrm{~mL})$ was removed by pipetting, and the precipitate $(0.86 \mathrm{~mL})$ was collected as the hydrogel of TiNS in the repulsion-dominant state (TiNS-Gel Repuls $_{\text {) }}$. Because the amount of TiNS included in the supernatant was negligibly small, the TiNS content in the resultant gel was calculated to $14 \mathrm{wt} \%$. TiNS-Gel $\mathrm{R}_{\text {Repuls }}$ doped with AuNPs was prepared in the similar procedure, except for adding an aqueous dispersion of AuNPs ([AuNP] $=$ $0.064 \mathrm{wt} \%, 0.67 \mathrm{~mL}$ ) to the TiNS dispersion ([TiNS] $=0.4 \mathrm{wt} \%, 30 \mathrm{~mL}$ ) before the centrifugation. Because the amount of AuNPs included in the supernatant was negligibly small, the AuNP content in the resultant gel was calculated to $0.05 \mathrm{wt} \%$.

Synthesis of gold nanoparticles (AuNPs). An aqueous dispersion of gold nanoparticles (AuNPs) were synthesized according to the literature ${ }^{33}$. Typically, a solution of $\mathrm{HAuCl}_{4} \cdot 3 \mathrm{H}_{2} \mathrm{O}$ in water $(0.01 \mathrm{wt} \%, 50 \mathrm{~mL})$ was heated to reflux. To the refluxing mixture, a solution of sodium citrate in water $(1.0 \mathrm{wt} \%, 1.0 \mathrm{~mL})$ was quickly injected. The color of the mixture turned to a brilliant red after $2 \mathrm{~min}$, and its reflux for additional $3 \mathrm{~min}$ afforded an aqueous dispersion of AuNPs. Thus obtained AuNPs were monodisperse with an average diameter of $17 \mathrm{~nm}$, as 
a
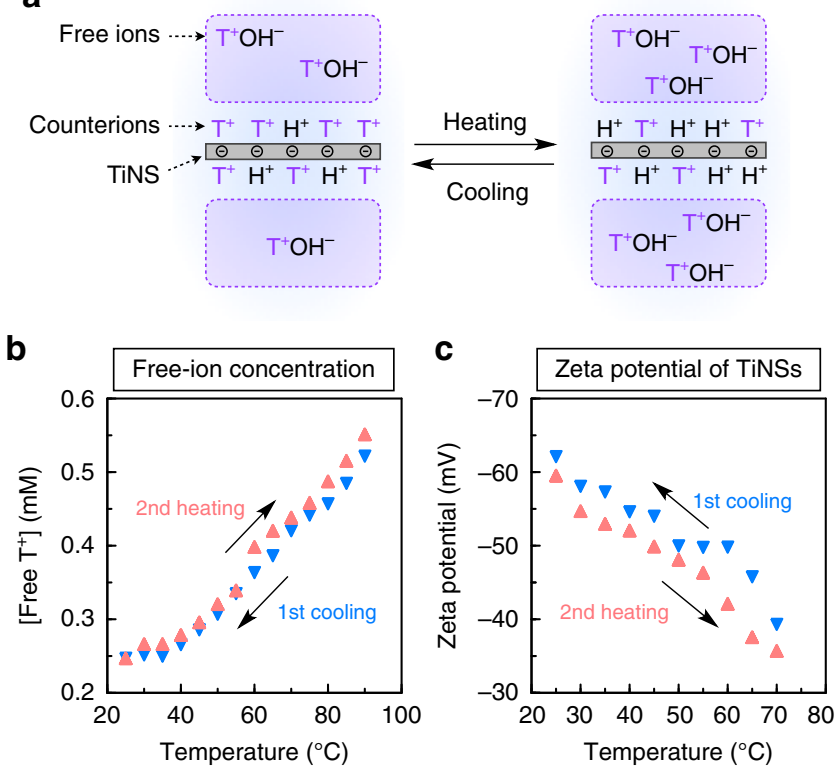

d

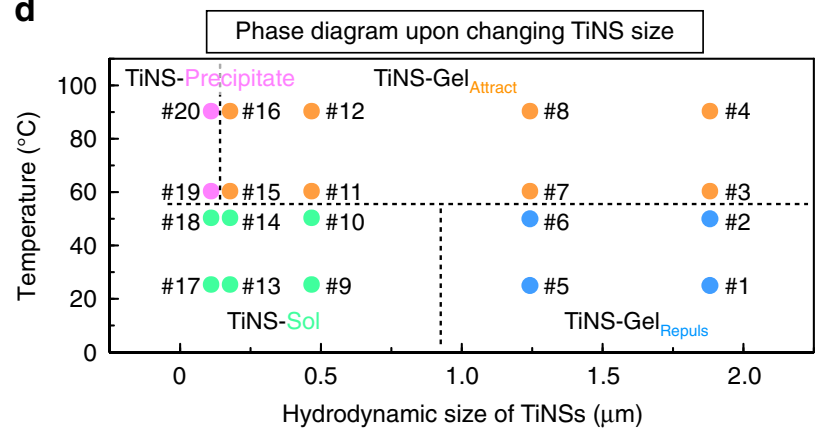

Fig. 6 Mechanism of gel-to-gel transition and phase diagram of TiNS-Gel.

a Schematic illustration for the mechanism of tunable electrostatic repulsion of TiNSs. TiNSs carry a lot of surface anions $\left(\mathrm{Ti}^{-} \mathrm{O}^{-}\right)$that form ion pairs with tetrabutylammonium countercations $\left(\mathrm{T}^{+}\right)$and protons $\left(\mathrm{H}^{+}\right)$. Upon heating, the countercations $\mathrm{T}^{+}$, which are originally trapped by $\mathrm{Ti}^{-} \mathrm{O}^{-}$ on TiNSs, are replaced with $\mathrm{H}^{+}$from bulk water. This thermally induced $\mathrm{T}$ $+/ \mathrm{H}^{+}$replacement increases the concentration of free $\mathrm{T}^{+}$ions and causes neutralization of the negative charges on $\mathrm{Ti}^{-} \mathrm{O}^{-}$on $\mathrm{TiNSs}$. Consequently, upon heating, the electrostatic repulsion between TiNSs is attenuated. Upon cooling, the opposite changes occur, so that the electrostatic repulsion between TiNSs is recovered. $\mathbf{b}, \mathbf{c}$ Changes in the concentration of free ions of $\mathrm{T}^{+}$(measured for a $0.1 \mathrm{wt} \%$ TiNS dispersion; b) and surface potential of TiNS (measured for a 0.01 wt\% TiNS dispersion; c) upon thermal scanning. $\mathbf{d}$ Thermal phase diagram of TiNSs dispersed in water $([\mathrm{TiNS}]=14 \mathrm{wt} \%)$ with systematically changing the hydrodynamic size of TiNSs.

confirmed by TEM. Because AuNPs obtained in the above procedure could lose their colloidal dispersibility in the presence of TiNSs, AuNPs were coated with a polyethylene glycol ligand. Typically, thiol-appended polyethylene glycol monomethyl ether (average molecular weight: $6 \mathrm{kDa} ; 120 \mathrm{mg}$ ) was added to an aqueous dispersion of AuNPs ([AuNP] $=0.005 \mathrm{wt} \% ; 40 \mathrm{~mL}$ ), and the mixture was mechanically shaken at $25^{\circ} \mathrm{C}$ for $24 \mathrm{~h}$. For removal of the unreacted reagent, the resultant mixture was subjected to repetitive centrifugation at $20,000 \times g$ and subsequent re-dispersion with water to afford an aqueous dispersion of polyethylene glycol-coated AuNPs.

Small-angle X-ray scattering (SAXS) analysis. Two-dimensional SAXS measurements were carried out at BL45XU in the SPring-8 synchrotron radiation facility (Hyogo, Japan) ${ }^{44}$ using Dectris model PILATUS3X $2 \mathrm{M}$ detectors. The scattering vector $q(q=4 \pi \sin \theta / \lambda ; 2 \theta$ and $\lambda=$ scattering angle and wavelength of an incident X-ray beam [1.0 $\mathrm{A}$, respectively) and position of an incident X-ray beam on the detector were calibrated using several orders of layer reflections from silver behenate ( $d=58.380 \AA$ ). The sample-to-detector distance was $2.5 \mathrm{~m}$, where acquired scattering $2 \mathrm{D}$ images were integrated along the Debye-Scherrer ring, affording the corresponding one-dimensional profiles. A TiNS hydrogel sample held in a glass capillary $(1.5 \mathrm{~mm}$ in diameter) was measured with gradually changing the temperature in a range of $25-90^{\circ} \mathrm{C}$ (Supplementary Fig. 3).

Rheological tests. Rheological tests of TiNS gel were carried out by using an Anton Paar model MCR-301 rheometer. A hydrogel sample was set under a 25$\mathrm{mm}$ diameter parallel plate with a sample gap of $1.0 \mathrm{~mm}$. Storage and loss moduli $\left(G^{\prime}\right.$ and $G^{\prime \prime}$, respectively) were measured (i) on frequency sweep in a range of $0.1-10 \mathrm{rad} \mathrm{s}^{-1}$ at a fixed strain of $0.1 \%$ with keeping the temperature at $25^{\circ} \mathrm{C}$ (Fig. 4a and Supplementary Fig. 5b) and $90^{\circ} \mathrm{C}$ (Fig. $4 \mathrm{~b}$ and Supplementary Fig. 5c) and (ii) at a fixed frequency of $1.0 \mathrm{rad} \mathrm{s}^{-1}$ and a fixed strain of $0.1 \%$ with changing the temperature in a range of a range of $25-90^{\circ} \mathrm{C}$ at a rate of $1{ }^{\circ} \mathrm{C} \mathrm{min}-1$ (Fig. 4c).

Compression tests. Compression tests of TiNS gel were carried out by using the same machine and set up as described for the rheological tests. Compressive stress was measured with gradually decreasing the sample gap from 1.0 to $0.99 \mathrm{~mm}$ at a rate of $0.02 \mathrm{~mm} \mathrm{~min}^{-1}$ with keeping the temperature at $25^{\circ} \mathrm{C}$ (Supplementary Fig. 4a) and $90^{\circ} \mathrm{C}$ (Supplementary Fig. $4 \mathrm{~b}$ ).

\section{Data availability}

The authors declare that the data supporting the findings of this study are available within the paper and its supplementary information files. All other information is available from the corresponding authors upon reasonable request.

Received: 20 June 2020; Accepted: 27 October 2020; Published online: 27 November 2020

\section{References}

1. Aida, T., Meijer, E. W. \& Stupp, S. I. Functional supramolecular polymers. Science 335, 813-817 (2012).

2. Webber, M. J., Appel, E. A., Meijer, E. W. \& Langer, R. Supramolecular biomaterials. Nat. Mater. 15, 13-26 (2016).

3. Zhang, Y. S. \& Khademhosseini, A. Advances in engineering hydrogels. Science 356, eaaf3627 (2017).

4. Sano, K., Ishida, Y. \& Aida, T. Synthesis of anisotropic hydrogels and their applications. Angew. Chem. Int. Ed. 57, 2532-2543 (2018).

5. Palagi, S. \& Fischer, P. Bioinspired microrobots. Nat. Rev. Mater. 3, 113-124 (2018).

6. Prince, E. \& Kumacheva, E. Design and applications of man-made biomimetic fibrillar hydrogels. Nat. Rev. Mater. 4, 99-115 (2019).

7. Shanmuganathan, K., Capadona, J. R., Rowan, S. J. \& Weder, C. Biomimetic mechanically adaptive nanocomposites. Prog. Polym. Sci. 35, 212-222 (2010).

8. Montero De Espinosa, L., Meesorn, W., Moatsou, D. \& Weder, C. Bioinspired polymer systems with stimuli-responsive mechanical properties. Chem. Rev. 117, 12851-12892 (2017).

9. Capadona, J. R., Shanmuganathan, K., Tyler, D. J., Rowan, S. J. \& Weder, C. Stimuli-responsive polymer nanocomposites inspired by the sea cucumber dermis. Science 319, 1370-1374 (2008).

10. Kouwer, P. H. J. et al. Responsive biomimetic networks from polyisocyanopeptide hydrogels. Nature 493, 651-655 (2013).

11. Cudjoe, E. et al. Biomimetic reversible heat-stiffening polymer nanocomposites. ACS Cent. Sci. 3, 886-894 (2017).

12. Fernańdez-Castaño Romera, M. et al. Mimicking active biopolymer structures with a synthetic hydrogel. J. Am. Chem. Soc. 141, 1989-1997 (2019).

13. De Almeida, P. et al. Cytoskeletal stiffening in synthetic hydrogels. Nat. Commun. 10, 609 (2019).

14. Palmer, L. C., Newcomb, C. J., Kaltz, S. R., Spoerke, E. D. \& Stupp, S. I. Biomimetic systems for hydroxyapatite mineralization inspired by bone and enamel. Chem. Rev. 108, 4754-4783 (2008).

15. Studart, A. R. Towards high-performance bioinspired composites. Adv. Mater. 24, 5024-5044 (2012).

16. Wegst, U. G. K., Bai, H., Saiz, E., Tomsia, A. P. \& Ritchie, R. O. Bioinspired structural materials. Nat. Mater. 14, 23-36 (2015).

17. Grzelczak, M., Liz-Marzan, L. M. \& Klajn, R. Stimuli-responsive self-assembly of nanoparticles. Chem. Soc. Rev. 48, 1342-1361 (2019).

18. Yu, A. C. et al. Scalable manufacturing of biomimetic moldable hydrogels for industrial applications. Proc. Natl Acad. Sci. USA 113, 14255-14260 (2016).

19. Feinle, A., Elsaesser, M. S. \& Hüsing, N. Sol-gel synthesis of monolithic materials with hierarchical porosity. Chem. Soc. Rev. 45, 3377-3399 (2016).

20. Diba, M., Wang, H., Kodger, T. E., Parsa, S. \& Leeuwenburgh, S. C. G. Highly elastic and self-healing composite colloidal gels. Adv. Mater. 29, 1604672 (2017). 
21. Nagai, N. et al. Fabrication of boehmite and $\mathrm{Al}_{2} \mathrm{O}_{3}$ nonwovens from boehmite nanofibres and their potential as the sorbent. J. Mater. Chem. 22, 21225-21231 (2012).

22. Cummins, H. Z. Liquid, glass, gel: the phases of colloidal laponite. J. NonCryst. Solids 353, 3891-3905 (2007).

23. Michot, L. J. et al. Liquid-crystalline aqueous clay suspensions. Proc. Natl Acad. Sci. USA 103, 16101-16104 (2006).

24. Mourad, M. C. D., Byelov, D. V., Petukhov, A. V. \& Lekkerkerker, H. N. W. Structure of the repulsive gel/glass in suspensions of charged colloidal platelets. J. Phys.: Condens. Matter 20, 494201 (2008).

25. Lekkerkerker, H. N. W. \& Vroege, G. J. Liquid crystal phase transitions in suspensions of mineral colloids: new life from old roots. Philos. Trans. A Math. Phys. Eng. Sci. 371, 20120263 (2013).

26. Verwey, E. J. W. \& Overbeek, J. T. G. Theory of the Stability of Lyophobic Colloids (Elsevier, 1948).

27. Sano, K. et al. Photonic water dynamically responsive to external stimuli. Nat. Commun. 7, 12559 (2016).

28. Sasaki, T., Watanabe, M., Hashizume, H., Yamada, H. \& Nakazawa, H. Macromolecule-like aspects for a colloidal suspension of an exfoliated titanate. Pairwise association of nanosheets and dynamic reassembling process initiated from it. J. Am. Chem. Soc. 118, 8329-8335 (1996).

29. Sano, K. et al. Extra-large mechanical anisotropy of a hydrogel with maximized electrostatic repulsion between cofacially aligned 2D electrolytes. Angew. Chem. Int. Ed. 57, 12508-12513 (2018).

30. Sano, K. et al. Internal structure and mechanical property of an anisotropic hydrogel with electrostatic repulsion between nanosheets. Polymer 177, 43-48 (2019).

31. Thurmond, F. A. \& Trotter, J. A. Morphology and biomechanics of the microfibrillar network of sea cucumber dermis. J. Exp. Biol. 199, 1817-1828 (1996).

32. Motokawa, T. \& Tsuchi, A. Dynamic mechanical properties of body-wall dermis in various mechanical states and their implications for the behavior of sea cucumbers. Biol. Bull. 205, 261-275 (2003).

33. Sun, Z. et al. An anisotropic hydrogel actuator enabling earthworm-like directed peristaltic crawling. Angew. Chem. Int. Ed. 57, 15772-15776 (2018).

34. Iwaso, K., Takashima, Y. \& Harada, A. Fast response dry-type artificial molecular muscles with [c2] daisy chains. Nat. Chem. 8, 625-632 (2016).

35. Chen, J. et al. Artificial muscle-like function from hierarchical supramolecular assembly of photoresponsive molecular motors. Nat. Chem. 10, 132-138 (2018).

36. Li, L., Scheiger, J. M. \& Levkin, P. A. Design and applications of photoresponsive hydrogels. Adv. Mater. 31, 1807333 (2019).

37. $\mathrm{Gu}, \mathrm{Y}$. et al. Photoswitching topology in polymer networks with metal-organic cages as crosslinks. Nature 560, 65-69 (2018).

38. Zhao, D. et al. A dynamic gel with reversible and tunable topological networks and performances. Matter 2, 390-403 (2020).

39. Pertsin, A. \& Grunze, M. Temperature dependence of the short-range repulsion between hydrated phospholipid membranes: a computer simulation study. Biointerphases 2, 105-108 (2007).

40. Saunders, A. E., Ghezelbash, A., Smilgies, D. M., Sigman, M. B. \& Korgel, B. A. Columnar self-assembly of colloidal nanodisks. Nano Lett. 6, 2959-2963 (2006).

41. Jana, S., Davidson, P. \& Abécassis, B. CdSe nanoplatelets: living polymers. Angew. Chem. Int. Ed. 128, 9517-9520 (2016).

42. Roy, D., Brooks, W. L. \& Sumerlin, B. S. New directions in thermoresponsive polymers. Chem. Soc. Rev. 42, 7214-7243 (2013).

43. Wei, M., Gao, Y., Li, X. \& Serpe, M. J. Stimuli-responsive polymers and their applications. Polym. Chem. 8, 127-143 (2017).
44. Fujisawa, T. et al. Small-angle X-ray scattering station at the SPring-8 RIKEN beamline. J. Appl. Crystallogr. 33, 797-800 (2000).

\section{Acknowledgements}

This work was financially supported by a JSPS Grant-in-Aid for Scientific Research (S) (18H05260) and a JSPS Grant-in-Aid for Scientific Research (B) (20H02791). We also acknowledge JST CREST Grant Number JPMJCR17N1, Japan. K.S. thanks JSPS for Grant-in-Aid for Research Activity Start-up (19K23642) and Young Scientists (20K15350) and Kurita Water and Environment Foundation (KWEF, Japan). K.S. also acknowledges RIKEN for the Special Postdoctoral Researcher Program. The small-angle $\mathrm{X}$-ray scattering measurements were performed at BL45XU in SPring-8 with the approval of the RIKEN SPring-8 Center (proposal 20180067).

\section{Author contributions}

K.S. conceived the project and designed the experiments. K.S. and N.I. performed all experiments. T.A. and Y.I. co-designed the experiments. Y.E. and T.S. prepared colloidally dispersed TiNSs. T.H. supported the small-angle X-ray scattering measurements at SPring-8. K.S. and Y.I. analyzed the data and wrote the manuscript with the input of all other authors. The manuscript reflects the contributions of all authors.

\section{Competing interests}

The authors declare no competing interests.

\section{Additional information}

Supplementary information is available for this paper at https://doi.org/10.1038/s41467020-19905-4.

Correspondence and requests for materials should be addressed to K.S., T.A. or Y.I.

Peer review information Nature Communications thanks Subi George, Myongsoo Lee and the other, anonymous, reviewer(s) for their contribution to the peer review of this work. Peer reviewer reports are available.

Reprints and permission information is available at http://www.nature.com/reprints

Publisher's note Springer Nature remains neutral with regard to jurisdictional claims in published maps and institutional affiliations.

(c) (i) Open Access This article is licensed under a Creative Commons Attribution 4.0 International License, which permits use, sharing, adaptation, distribution and reproduction in any medium or format, as long as you give appropriate credit to the original author(s) and the source, provide a link to the Creative Commons license, and indicate if changes were made. The images or other third party material in this article are included in the article's Creative Commons license, unless indicated otherwise in a credit line to the material. If material is not included in the article's Creative Commons license and your intended use is not permitted by statutory regulation or exceeds the permitted use, you will need to obtain permission directly from the copyright holder. To view a copy of this license, visit http://creativecommons.org/ licenses/by/4.0/.

(c) The Author(s) 2020 